\title{
Aberration-Corrected STEM Imaging Through Off-Site Remote Operation
}

\author{
K. A. Jarvis, * Lawrence F. Allard,** Timothy Y. Jerome, ${ }^{* *}$ Thomas C. Isabell,*** \\ Douglas A. Blom, **** and Paulo J. Ferreira* \\ * Materials Science and Engineering Program, University of Texas at Austin, Austin, TX 78712 \\ ** Materials Science \& Technology Div., Oak Ridge National Laboratory, Oak Ridge, TN 37831 \\ *** TEM Product Division, JEOL USA Inc. Peabody, MA 01960 \\ **** EM Center, University of South Carolina, 715 Sumter St, Columbia, SC 29208
}

Recent advances in aberration-corrected electron microscopy have allowed researchers to image materials at sub-Ångström resolution. Many of these modern instruments are designed to be operated from separate 'control' rooms, removing the effect of the operator on the instrument's physical environment. This capability also allows operation from suitable workstations, over internet connections, from literally anywhere in the world [1]. Researchers at the University of Texas at Austin (UTA) have collaborated with Oak Ridge National Laboratory (ORNL) and JEOL Ltd. to routinely conduct research sessions in which high-resolution images and X-ray microanalytical data are acquired during after-hours research sessions, utilizing the JEOL 2200FS aberration-corrected STEM/TEM at ORNL from their lab in Austin. Details of the remote operation are presented here.

At the beginning of the session, a local ORNL operator loads the appropriate sample and ensures the anti-contamination cold finger reservoirs are full of liquid $\mathrm{N}_{2}$. The remainder of the session is controlled remotely. Virtually every microscope function is accessible from the remote workstation. There are four computers at ORNL that are used during local operation, one for JEOL TEM Controller Application 2.18 (TEMCON), one for controlling the corrector (CEOS GmbH CESCOR software), one to access Gatan's DigitalMicrograph ${ }^{\mathrm{TM}}$ (DM) software, and one to run the JEOL scan controller (ASID). Remote viewers are provided with an ORNL computing account and Cisco VPN Client v.5.0.03.0560. They also obtain a firewall exception with their static IP address that allows them to connect to ORNL local computers. The ORNL local computers run UltraVNC Server software to host a connection from the remote user's computer. Remote Access Client 3.5.0 (PCNetSoftware) runs on the DM computer as well. This allows the DM computer to provide remote desktop viewing of the JEOL TEMCON to remote viewers concomitantly. The remote user then makes a connection to the ORNL LAN via Cisco VPN and inputs their ORNL computing user name and password. With a Java-enabled web browser and ORNL-provided web address and temporary password, the user can load UltraVNC through the remote viewer's web browser. No additional software is required on the remote PC. The remote viewer now has a webpage loaded that contains an extended desktop view of two computer windows, allowing full control of both the TEMCON and the DM computers. In place of the control knob set used at ORNL, Prof. Ferreira's lab uses JEOL's Sirius package, which includes Sirius Client 2.0.1.0 software, a trackball for all stage movement and tilts, and a "reduced" knob set for TEM control. The ASID computer at ORNL is not accessed remotely, as DM provides suitable fast scanning functions that are used instead. All imaging set-ups can be conducted remotely. The Sirius graphical user interface (GUI) along with the trackball is used to, e.g., open the gun valve, translate and tilt the sample, and adjust the specimen to the proper Z-height. Beam modes (e.g. MAG for imaging and AMAG for EDS analysis) are set up using the scanning control panel window on the TEMCON screen. Beam tilt and condenser stigmation are trimmed via a Ronchigram, using the Sirius GUI, the remote knob set and the high-speed camera image on TEMCON. For imaging, a condenser aperture is inserted and centered using Sirius, and the BF and HAADF detectors appropriately activated. Figure 1 shows 
simultaneous views of the control room at the JEOL 2200FS laboratory with Dr. Larry Allard present, watching the operation by Dr. Karalee Jarvis, with Prof. Paulo Ferreira observing in their laboratory at UTA. Figure 2 is an example of an imaging result; a HAADF/STEM image is shown at $8 \mathrm{Mx}$ direct magnification of Pt particles on a carbon film, with clearly defined atomic columns and single atoms seen (arrows), recorded from 1400km away. Other available techniques, such as EDS analysis and spectrum imaging, and electron energy-loss spectroscopy, can also be conducted remotely, with limited assistance at times from an on-site operator. Figure 3 is a screen shot showing imaging and EDS results from a recent session. Remote microscopy in its fullest sense is now a reality, and contributes to more efficient use of a multi-million-dollar instrument [2].

\section{References}

[1] J.M. Perkins, et al., Micros \& Microanal (2007), 13 (Suppl. 02) : 1702-1703

[2] This research at the Oak Ridge National Laboratory's High Temperature Materials Laboratory was sponsored by the U. S. Department of Energy, Office of Energy Efficiency and Renewable Energy, Vehicle Technologies program.

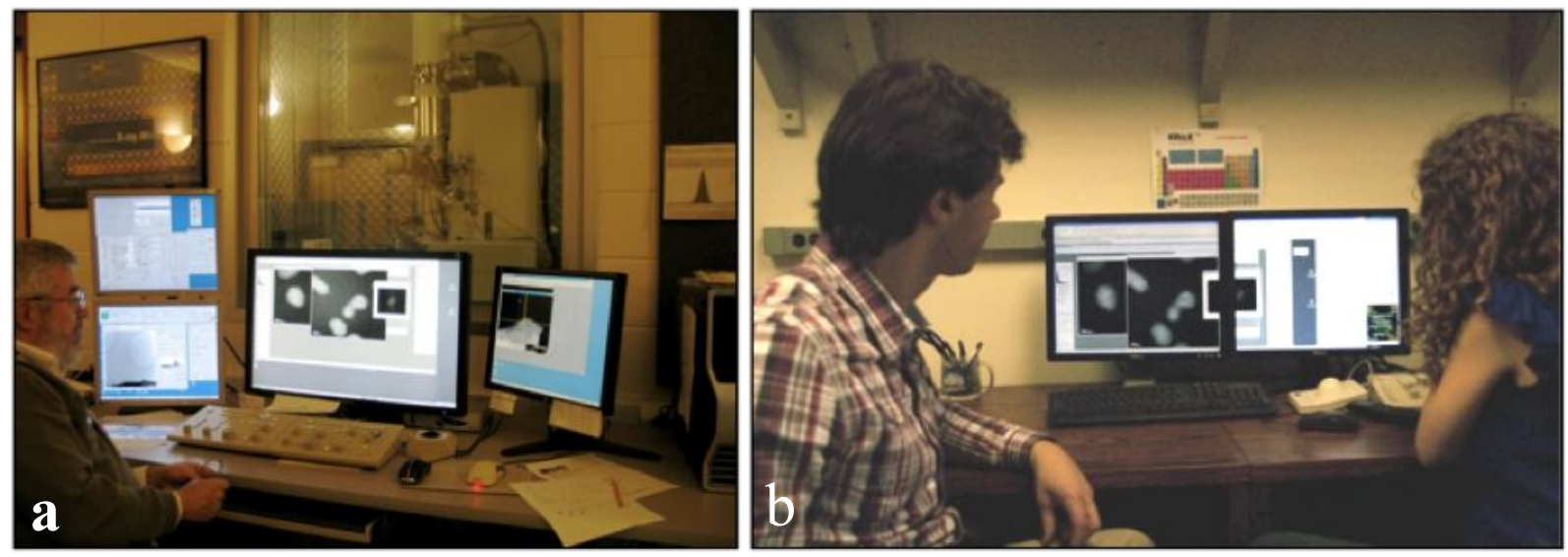

FIG 1. Image taken during a remote control session from a) the JEOL 2200FS control room at Oak Ridge National Laboratory and b) Prof. Ferreira's lab at the University of Texas at Austin. The microscope can be seen through the window in image (a).
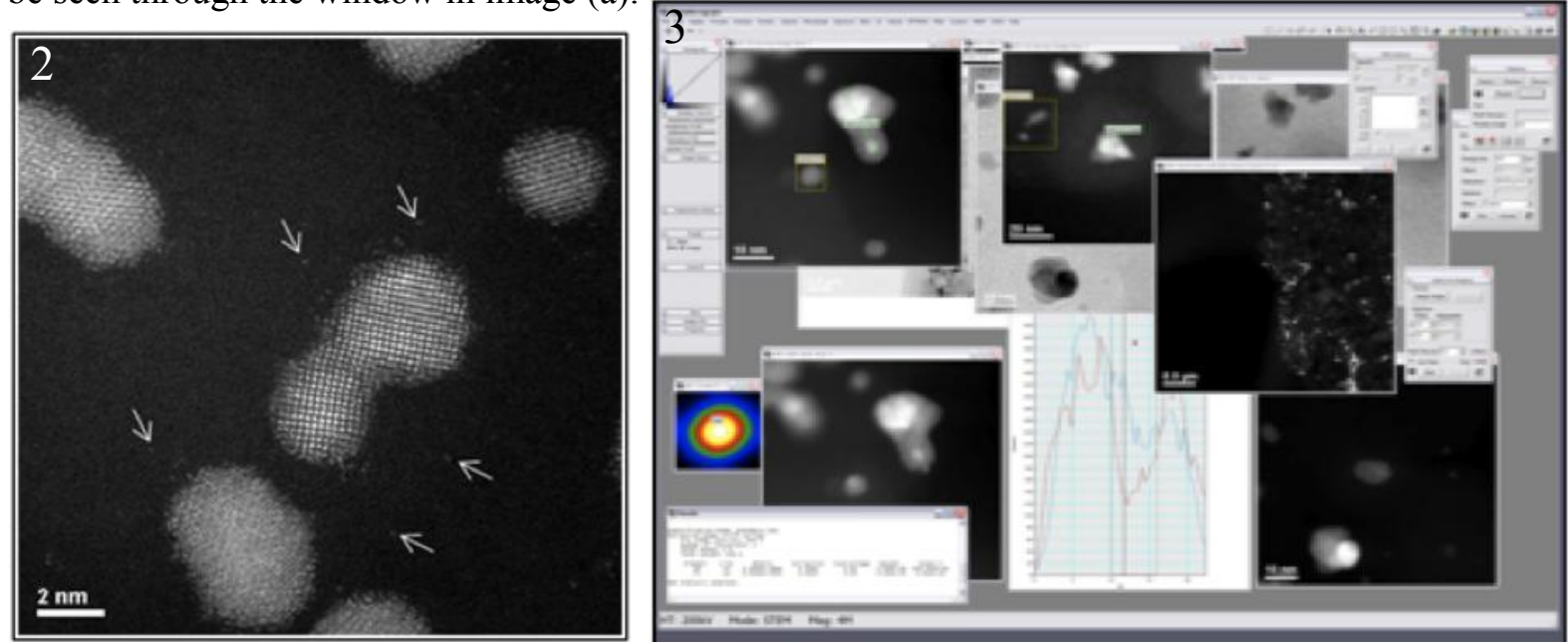

FIG. 2. Cs-corrected HAADF/STEM images of Pt nanoparticles on a carbon support recorded at $8 \mathrm{Mx}$ direct magnification. The (200) interplanar spacing of $\mathrm{Pt}(1.96 \AA)$ and single $\mathrm{Pt}$ atoms (arrows) are seen.

FIG. 3. Screen shot from DM computer, showing some multi-technique results obtained during a lateevening remote operation session from UTA. 